\title{
OWL-DL as a Power Tool to Model Negotiation Mechanisms with Incomplete Information
}

\author{
Azzurra Ragone \\ SisInfLab, Politecnico di Bari, I-70125, Bari, Italy \\ a.ragone@poliba.it
}

\begin{abstract}
We propose a logic-based framework to model negotiations among agents in scenarios with fully incomplete information. In particular, we address multi-issue bilateral negotiations, with issues are expressed and related to each other via an OWL ontology. In particular we use OWL-DL sub-language and exploit its formal semantics, based on $\mathcal{S H O I N}(D)$. Agents goals are expressed through OWL-DL as (complex) concept expressions, and the worth of goals as weights over concepts. Noteworthily, we adopt a very general setting with incomplete information by letting agents keep both goals and worths of goals as private information. Moreover we propose a protocol for agents to negotiate on and reach an agreement, exploiting inference services. Agents, according to the protocol, can pursue different sets of strategies — depending on their type — and we illustrate and motivate two possible strategies.
\end{abstract}

\section{Introduction}

Several recent research efforts have been focused on automated negotiation in various contexts, including e-marketplaces, resource allocation settings, online auctions, supply chain management and, in general terms, e-business processes. Negotiation mechanisms usually model resource and task allocation problems where issues to negotiate on are well established and defined in advance, e.g., online auctions. Many other negotiation mechanisms instead model e-marketplaces of undifferentiated products (commodities) where the only issues to negotiate on are price or quantity. Nevertheless there a number of frameworks where agents have to reach an agreement on a product (car, house, etc.) or service (travel booking, wedding service, etc.) that can be described by many issues amenable to negotiation, and such issues may well be not necessarily all established in advance. Moreover Buyer (Requester) and Seller (Provider) may be not necessarily interested in the same set of issues and may have different preferences on bundles of interrelated issues. Obviously, if issues are not fixed there is the problem to express what agents "want" or "prefer". For instance, considering an housing scenario, how to express a request for a double room within an apartment provided with a washing machine for a period of six month or, conversely an offer for single or double room in an apartment where pets are not allowed and the bills are included in the lease? Is there any negotiation space? Can an agreement — in an automated way_ be reached? Trying to answer to this and other questions, we present a framework for multi-issue bilateral negotiation, with issues expressed and related to each other exploiting OWL-DL formalism. Agents goals are expressed through (complex) concepts, and the worth of goals as 
weights over concepts. We consider a very general setting with incomplete information, i.e., agents keep both goals and worths of goals as private information. We introduce a negotiation protocol and possible agents' strategies for such a setting, exploiting inference services-satisfiability and subsumption-available as services in DL reasoners. In a nutshell, satisfiability can be useful to catch inconsistency between agent's goals w.r.t. the ontology $\mathcal{T}$, i.e., inconsistent goals cannot be in the same agreement, (e.g., agents cannot agree on $A$ and $B$ at the same time if in $\mathcal{T} A$ is defined as disjoint from $B)$. Through subsumption one can discover if an agent's goal is implied by a goal of its opponent, even if this fact does not immediately appear at the syntactic level.

\section{Scenario}

We set the stage of our approach in a generic framework with incomplete information: we assume agents do not reveal their goals either to the opponent or to a mediator, but they keep as private information both goals and their worths. Actually, the difficulty to model scenarios with incomplete information is due to the fact that the agent cannot be sure how the opponent will evaluate its offers, and therefore it may be unable to negotiate to the best of its capacity [4]. Usually, to overcome such drawback, a preliminary step is added to strategic negotiations where agents reveal some (or all) private information. Obviously, the revelation mechanism has to ensure agents truthfully report their private information and punish liars [12]. Yet it is not always possible to design truthful revelation mechanisms, since they depend on the particular scenario taken into account (see [4, p.64] for an extensive discussion). When negotiation involves organizations, e.g., companies, revealing information may conflict with company's interests and assessing the truthfulness of the agents' declarations can can be very hard or impossible. An advantage of our proposal is that having considered a "worst case" scenario, we can then —in a principled way — study simplified negotiation scenarios.

The protocol we propose here is able to deal with such incomplete information without forcing agents to reveal neither their goals nor thier utility functions, so it suits all scenarios where agents are not willing to reveal private information or when it is hard to design a truthful revelation mechanism. In this paper we refer, as an example, to a scenario where agents negotiate for a lease. Let us suppose that Amy is a student having to spend a period of about six months abroad as visiting student, so she needs to find a room in an apartment and she has to negotiate with the landlord about the lease terms. She is searching for a bedroom, which is not a multiple room, in an apartment provided with washing machine; actually she would prefer a single room, but this is not a mandatory constraint. She is looking for an apartment where cats are not allowed, because of her allergy, and where no security deposit is required and bills are included; finally she would prefer to have a parking (street parking or own parking) near the apartment. The following goals can be formalized as in Fig. 11 On the other hand also the landlordOscar-has some goals, that can be formalized as in Fig. 2. Relations among issue are expressed in the Ontology reported in Fig. 3. Obviously, the one described above is not the only feasible scenario to apply the approach proposed, since the negotiation framework we propose is very general and can be applied to several negotiation scenarios where resource descriptions can be modeled through KR languages. 


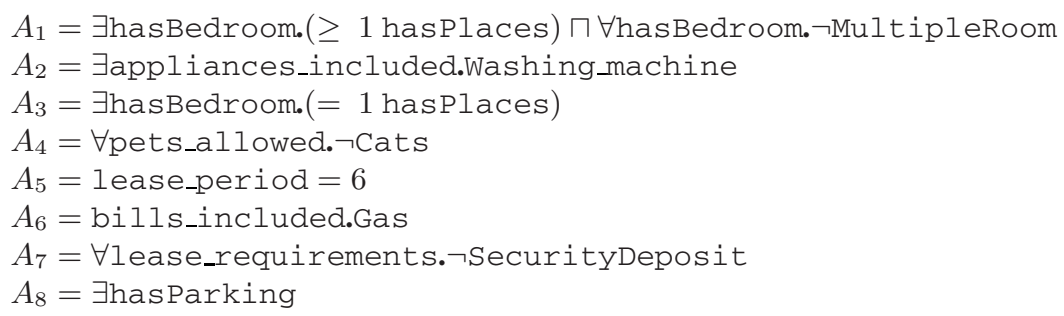

Fig. 1. Amy's goals formalized using the logic $\mathcal{S H O} \mathcal{I N}(D)$

$O_{1}=\exists$ hasBedroom.DoubleRoom

$\mathrm{O}_{2}=\exists$ has SharedRoom.Laundry

$\mathrm{O}_{3}=\neg \exists$ pets_allowed

$\mathrm{O}_{4}=$ lease_period_negotiable

$O_{5}=\forall$ bills_included. $\perp$

$O_{6}=\exists$ lease_requirements.SecurityDeposit

$\mathrm{O}_{7}=\forall$ hasParking.StreetParking $\sqcap \exists$ hasParking

Fig. 2. Oscar's goals formalized using the logic $\mathcal{S H O I N}(D)$

$\mathcal{T}$

SingleRoom $\equiv$ Bedroom $\sqcap(=1$ hasPlaces $)$

DoubleRoom $\equiv$ Bedroom $\sqcap$ (= 2 hasPlaces $)$

MultipleRoom $\equiv$ Bedroom $\sqcap(\geq 3$ hasPlaces $)$

Laundry $\sqsubseteq$ Room $\sqcap \exists$ appliances_included.(Washing_machine $\sqcap$ Dryer)

$\exists$ hasRoom.Laundry $\sqsubseteq \exists$ appliances_included.(Washing_machine $\sqcap$ Dryer)

has SharedRoom $\sqsubseteq$ hasRoom

hasBedroom $\sqsubseteq$ hasRoom

lease_period_negotiable $\equiv$ lease_period $\leq 12$

Bills $\equiv$ ONE-OF\{Gas, Electricity, Water

House $\equiv \exists$ hasBedroom $\sqcap$ ᄏhasRoom.Bathroom

Fig. 3. The Ontology used in the example

\section{The Negotiation Protocol}

Following the idea in [5] agents negotiate making proposals and counter-proposals. At each round the agent can decide to accept the proposal made by its opponent, to reject such a proposal or to refine the proposal itself, until an agreement is reached or an agent quits the negotiation. In order to define the protocol we define the utility of $a$ proposal and a motivated proposal. A motivated proposal is a proposal such that 1 . it is not in conflict with what has been agreed in the previous rounds of the protocol: 2 . it increases the utility of the agent making it. We call such a protocol Cumulative protocol because the agreement is reached in an incremental way, adding at each round a goal or a set of goals, which can be satisfiable w.r.t. the ontology. It is possible to demonstrate that such a protocol converges. Given the rules of the protocol it is possible to pursue different strategies. We analyzed the properties of two strategies, the IWIN strategy and 
CoCo strategy. The former aims at maximizing in the first step the utility of the agent making the first proposal; although we demonstrated that this strategy allows to reach Pareto-efficient agreements, it may yield to highly unfair distributions of utilities in a proposal, hence, a negotiation where both agents immediately saturate their utility is likely to be a very long sequence of proposals and rejections. Therefore we introduced another strategy called CoCo, where agents try to compensate the loss in utility when they accept a proposal made by the opponent. Since the CoCo strategy uses a greedy approach the final agreement might not be Pareto-efficient, yet a computational analysis has clearly shown that CoCo has a low computational effort compared to IWIN1.

\section{Relations with Related Work and Conclusion}

Automated bilateral negotiation between agents has been widely investigated, both in artificial intelligence and in microeconomics research communities. AI-oriented research has usually focused on automated negotiation between agents and on designing high-level protocols for agent interaction. Agents can play different roles: act on behalf of buyer or seller, but also play the role of a mediator or facilitator. In the following we give a brief overview of logic-based approaches to automated negotiation, comparing our approach with existing ones and highlighting differences. In [1] the use of propositional logic in multi-issue negotiation was investigated, while in [2] weighted propositional formulas in preference modeling were considered. However, in such works, no semantic relation between issues is taken into account. In our approach we adopt a logical theory, i.e., an ontology, which allows one e.g., to catch inconsistencies between demand and supply, model implication, find out a feasible agreement in a bundle, which are fundamental issues to model in a negotiation setting. In [13] an agreement is defined as a model for a set of formulas from both agents. The approach does not take preferences into account, so that it is not possible to guarantee that the reached agreement is Pareto-efficient. With reference to the work presented in [14], adopting a propositional logic setting, common knowledge is considered as just more entrenched preferences, that could be even dropped in some deals. We adopt a knowledge base, or ontology $\mathcal{T}$, of formulas which are common knowledge for both agents, whose constraints must always be enforced in the negotiation outcomes. Moreover we use additive utilitites over formulas: this allows an agent to make compensations between its requests and its concessions, while in [14] the concession of a more entrenched formula can never be compensated by less entrenched ones, no matter how many they are. Finally we devised a protocol which the agents should adhere to while negotiating; in contrast in [14] a game-theoretic approach is taken, presenting no protocol at all, since communication between agents is not considered. Prior to considering description logics as logical language for bilateral negotiation, we have studied different negotiation mechanism with the presence of a mediator [7/611] and without a mediator with partial incomplete information [10], in the framework of theory-endowed propositional logic. To the best of our knowledge, our approach is the first one using DLs to design a logic-based negotiation mechanism, ensuring a greater expressiveness w.r.t. propositional logic. Moreover,

\footnotetext{
${ }^{1}$ For the sake of conciseness we omit a lot of details about the protocol and the evaluation of strategies, which can be found in [9].
} 
w.r.t. to non-logic-based approaches, the use of an ontology $\mathcal{T}$ allows exploiting inference services that are used in the actual negotiation mechanisms. In future work we are planning to validate our approach with agent-based simulations and we are also setting up an analysis of the game theoretic properties, as related properties of the negotiation protocols ( e.g., Pareto-efficiency), equilibrium strategies or properties of the agents (e.g., individual rationality). Research is also ongoing exploiting, in the negotiation mechanism, non-monotonic inferences in DLs [3]-namely Concept Contraction - to identify and manage conflicting information [8].

\section{References}

1. Bouveret, S., Lemaitre, M., Fargier, H., Lang, J.: Allocation of indivisible goods: a general model and some complexity results. In: Proc. of AAMAS 2005, pp. 1309-1310 (2005)

2. Chevaleyre, Y., Endriss, U., Lang, J.: Expressive power of weighted propositional formulas for cardinal preference modeling. In: Proc. of KR 2006, pp. 145-152 (2006)

3. Di Noia, T., Di Sciascio, E., Donini, F.M.: Semantic Matchmaking as Non-Monotonic Reasoning: A Description Logic Approach. JAIR 29, 269-307 (2007)

4. Kraus, S.: Strategic Negotiation in Multiagent Environments. MIT Press, Cambridge (2001)

5. Parsons, S., Sierra, C., Jennings, N.: Agents that reason and negotiate by arguing. J. of Log. and Comp. 8(3), 261-292 (1998)

6. Ragone, A., Di Noia, T., Di Sciascio, E., Donini, F.M.: A logic-based framework to compute pareto agreements in one-shot bilateral negotiation. In: Proc. of ECAI 2006, pp. 230-234 (2006)

7. Ragone, A., Di Noia, T., Di Sciascio, E., Donini, F.M.: Propositional- logic approach to oneshot multi issue bilateral negotiation. ACM SIGecom Exchanges 5(5), 11-21 (2006)

8. Ragone, A., Di Noia, T., Di Sciascio, E., Donini, F.M.: Alternating-offers protocol for multiissue bilateral negotiation in semantic-enabled marketplaces. In: Proc. of ISWC 2007 (to appear, 2007)

9. Ragone, A., Di Noia, T., Di Sciascio, E., Donini, F.M.: Description logics for multi-issue bilateral negotiation with incomplete information. In: proc. of AAAI 2007, pp. 477-482. AAAI, Stanford, California, USA (2007)

10. Ragone, A., Di Noia, T., Di Sciascio, E., Donini, F.M.: Logic-based alternating-offers protocol for automated multi-issue bilateral negotiation in P2P e-marketplaces. In: Proc. of DL 2007 (2007)

11. Ragone, A., Di Noia, T., Di Sciascio, E., Donini, F.M.: When price is not enough: Combining logical and numerical issues in bilateral negotiation. In: proc. of AAMAS 2007, pp. 97-99. ACM Press, New York (2007)

12. Rosenschein, J.S., Zlotkin, G.: Rules of Encounter. MIT Press, Cambridge (1994)

13. Wooldridge, M., Parsons, S.: Languages for negotiation. In: Proc. of ECAI 2000, pp. 393 $400(2000)$

14. Zhang, D., Zhang, Y.: A computational model of logic-based negotiation. In: Proc. of AAAI 2006, pp. 728-733. AAAI, Stanford, California, USA (2006) 